\title{
Isolation of an NDM-5-producing ST16 Klebsiella pneumoniae from a Dutch patient without travel history abroad, August 2015
}

E Bathoorn ${ }^{1}$, JW Rossen ${ }^{1}$, M Lokate ${ }^{1}$, AW Friedrich ${ }^{1}$, AM Hammerum ${ }^{2}$

1. Department of Medical Microbiology, University of Groningen, University Medical Center Groningen, Groningen, the Netherlands

2. Department of Microbiology and Infection Control, Statens Serum Institut, Copenhagen, Denmark

Correspondence: Erik Bathoorn (d.bathoorn@umcg.nl)

Citation style for this article:

Bathoorn E, Rossen JW, Lokate M, Friedrich AW, Hammerum AM. Isolation of an NDM-5-producing ST16 Klebsiella pneumoniae from a Dutch patient without travel history abroad, August 2015. Euro Surveill. 2015;20(41):pii=30040. DOI: http://dx.doi.org/10.2807/1560-7917.ES.2015.20.41.30040

Article submitted on 02 October 2015 / accepted on 14 October 2015 / published on 15 October 2015

A New Delhi Metallo-beta-lactamase-5 (NDM-5)producing ST16 Klebsiella pneumoniae strain was isolated from a Dutch patient in a long-term care facility without recent travel history abroad. Core genome multilocus sequence typing (cgMLST) revealed that the Dutch isolate was clonally related to isolates detected in four patients in Denmark in 2014. Public health experts and clinicians need to be informed; repetitive screening may be needed in patients without known risk factors for carbapenemases-producing Enterobacteriaceae who have undergone antibiotic treatment.

Here we report of a New Delhi Metallo-beta-lactamase- 5 (NDM-5)-carrying ST16 Klebsiella pneumoniae, isolated from a hospitalised patient in the Netherlands, with no recent history of travel abroad. Analysis by core genome multilocus sequence typing (cgMLST) based on the core genome sequence of the isolate, showed that it is clonally related to four recently reported isolates cultured from four patients in two hospitals in Denmark, in 2014 [1].

\section{Background}

NDM is a carbapenemase that has been detected for the first time in a Swedish patient returning from New Delhi in 2008 [2]. In the first years thereafter, introduction of NDM-producing isolates in European hospitals was associated with returning travellers from India and Pakistan [3]. NDM-producing Enterobacteriaceae are rapidly dispersing over the world, and cases without any epidemiolgical links to the Indian subcontinent have been reported [4]. Reports on detection of NDM in environmental samples and in the food chain are worrisome, in particular since this might implicate spread of these resistance genes in the community $[5,6]$. In northern and western Europe, however, identification of patients with NDM-producing Enterobacteriaceae is still rare [7].

Currently, nine types of NDMs have been detected, of which NDM-1 is the most prevalent type [8]. NDM- 5 has been isolated for the first time in the United Kingdom in 2011 from a patient returning from India [9]. Cases of NDM-5-carrying Enterobacteriaceae are sporadic. Some unrelated cases have been described, for instance three cases in Algeria and a case in Spain, with no recent history of travel abroad $[10,11]$. The identification of unrelated cases colonised with the same of NDM-5producing $K$. pneumoniae ST16 clone in two countries with low prevalence, may be cause for concern.

\section{Case description}

A Dutch patient in their 50 s suffering from spinal cord injury had been admitted for rehabilitation in a long-term care facility in a northern region of the Netherlands. Upon admission in August 2015 (day o), swab cultures taken from throat and rectum were negative for carbapenem-resistant Gram-negatives. The patient was treated upon admission with ceftriaxone 2 gr once per day for seven days, for an infection.

On day 10, an NDM-5-producing $K$. pneumoniae was cultured from a rectal swab taken as part of routine screening. Antibiogram based on automated susceptibility testing (VITEK2, bioMerieux, Marcy l'Etoile, France) and E-tests (AB Biodisk, Mannheim, Germany) showed increased minimum inhibitory concentrations (MICs) to meropenem and imipenem, and susceptibility to gentamicin, fosfomycin, and colistin (Table).

Whole genome sequencing, de novo assembly, and assessment of multilocus sequence typing (MLST), cgMLST, and resistome were performed as described previously $[12,13]$. The strain harboured beta-lactam 


\section{FIGURE}

Minimal spanning tree of the four Danish NDM-5producing ST16 Klebsiella pneumoniae isolates, the Dutch isolate, and reference strain K. pneumoniae 1084

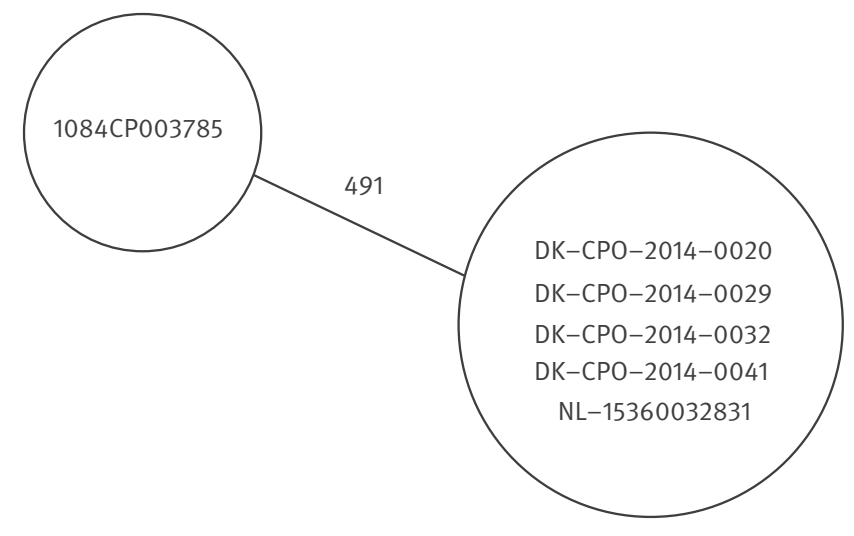

NDM: New Delhi Metallo-beta-lactamase.

Sequences of core genome genes of Danish (DK-) isolates, the Dutch (NL-) isolate, and reference strain K. pneumoniae 1084 (GenBank accession number CPo03785) were compared.

The tree is based on 634 columns, pairwise ignoring missing values. Number of allelic mismatches is presented.
TABLE

Antimicrobial minimum inhibitory concentrations for the NDM-5-producing Klebsiella pneumoniae isolate in a Dutch patient in a long-term care facility, August 2015

\begin{tabular}{|c|c|c|}
\hline Antimicrobial agent & $\mathrm{MIC}(\mathrm{mg} / \mathrm{L})^{\mathrm{a}}$ & Interpretation ${ }^{a}$ \\
\hline Piperacillin/tazobactam & $\geq 128$ & $S$ \\
\hline Cefotaxime $^{b}$ & 16 & $\mathrm{R}$ \\
\hline Ceftazidime & $\geq 64$ & $\mathrm{R}$ \\
\hline Cefepime & $\geq 64$ & $\mathrm{R}$ \\
\hline Meropenem $^{\mathrm{b}}$ & 6 & $\mathrm{I}$ \\
\hline Imipenem & $\geq 16$ & $\mathrm{R}$ \\
\hline Ciprofloxacin & $\geq 64$ & $\mathrm{R}$ \\
\hline Gentamicin & $\leq 1$ & $\mathrm{~S}$ \\
\hline Tobramycin & $\geq 64$ & $\mathrm{R}$ \\
\hline Trimethoprim-sulfamethoxazole & $\geq 320$ & $\mathrm{R}$ \\
\hline Colistin & $\leq 0.5$ & $\mathrm{~S}$ \\
\hline Tigecyclin $^{\mathrm{b}}$ & 2 & $\mathrm{I}$ \\
\hline Fosfomycin ${ }^{b}$ & 3 & $\mathrm{~S}$ \\
\hline
\end{tabular}

MIC: minimum inhibitory concentration; I: intermediate; R: resistant; S: susceptible.

according to the European Committee on Antimicrobial Susceptibility Testing (EUCAST) guidelines.

${ }^{\text {b }}$ Measured by E-test.

infection control guidelines recommend only to screen healthcare workers in case of ongoing transmission after implementation of outbreak control measures [14]. We did not identify any likely origins through these investigations.

Literature was searched for common sources of NDM5-producing Enterobacteriaceae. A recently published report on a Danish cluster of four patients with NDM5-producing $K$. pneumoniae clones presented a similar resistome to our case $\left(\right.$ bla $_{\mathrm{NDM}-5}$, bla $a_{\mathrm{CTX}-\mathrm{M}-15}, b l a_{\mathrm{SHV}-1}$, bla $a_{\mathrm{OXA}-1}$, and $\left.b / a_{\mathrm{TEM}-1 \mathrm{~b}}\right)$ [1]. Sequences of the Danish isolates were compared with the isolate from the Dutch case. All isolates belonged to ST16. The results of cgMLST analysis using a typing scheme described by Bialek-Davenet et al. [15] showed no allelic mismatches between our isolate and the Danish isolates (NCBI BioProject ID PRJNA285138). When comparing with reference strain K. pneumoniae 1084 (GenBank accession number (Po03785), we found 491 allelic mismatches (Figure). Thus, the isolate of our patient is clonally related to the Danish isolates.

\section{Discussion}

We report the sporadic detection of an NDM-5-producing K. pneumoniae from a Dutch patient with no risk factors for acquisition. The origin of the strain is unknown. Similar cases have been presented in Denmark with clonally related isolates. There were no known direct epidemiological links between the Danish patients and our case: the Danish positive cases did not travel to the Netherlands, and in our long-term care facility, no Danish patients were hospitalised during the past year. 
Unknown links or a common source may be the reason for the clonality of this rare isolate.

Another explanation might be that the clonal strains have been acquired from a foodborne source. Escherichia coli carrying bla $a_{\mathrm{NDM}-5}$, bla ${ }_{\mathrm{CTX}-\mathrm{M}}$ and bla ${ }_{\mathrm{TEM}}$ have been reported as cause of mastitis in cows, suggesting that bla $a_{\mathrm{NDM}-5}$-carrying plasmids might enter the food chain. Moreover, contamination of retail chicken meat with $K$. pneumoniae producing a combination of NDM, CTX-M-15, TEM, and SHV-1 has recently been reported [16]. Typing of bla ${ }_{\mathrm{NDM}}$, plasmid, and MLST of these isolates may reveal a link with a foodborne source.

K. pneumoniae ST16 is a highly prevalent type causing nosocomial infections [17]. It is unsure whether this ST16 clone is newly introduced, or whether this clone was already present in our region, and has now acquired an NDM-5-carrying plasmid. We anticipate a full study, which will assess the relation between the NDM-5-producing isolates and the epidemic CTX-M-15producing $K$. pneumoniae ST16 isolates in our region, and possible links with the food chain. Since our case was detected by chance and we did not identify any routes of transmission, further cases may be found in the Netherlands or elsewhere. We contacted national reference centers in Europe to find cases retrospectively, and to alert them about potential future cases. Finally, our case suggests that it might be necessary, under specific conditions, to screen for CPE also in patients who do not have a recent history of travel to a CPE-endemic country, and that diagnostics excluding CPE at admission, should be repeated if patients are using antibiotics during hospitalisation.

\section{Conflict of interest}

None declared.

\section{Authors' contributions}

EB collected the data and drafted the manuscript, JWR supervised the molecular research and analysis, ML organised infection control measurements and risk assessments, AWF participated in the coordination and concept of the manuscript, AMH coordinated and edited the manuscript.

\section{References}

1. Hammerum AM, Hansen F, Olesen B, Struve C, Holzknecht BJ, Andersen PS, et al. Investigation of a possible outbreak of NDM-5-producing ST16 Klebsiella pneumoniae among patients in Denmark with no history of recent travel using wholegenome sequencing. Journal of Global Antimicrobial Resistance 2015/09;3(3):219-221.

2. YongD, TolemanMA, GiskeCG, ChoHS, SundmanK, LeeK, et al. Characterization of a new metallo-beta-lactamase gene, bla(NDM-1), and a novel erythromycin esterase gene carried on a unique genetic structure in Klebsiella pneumoniae sequence type 14 from India. Antimicrob Agents Chemother. 2009;53(12):5046-54. DOI: 10.1128/AAC.00774-09 PMID: 19770275

3. KumarasamyK, KalyanasundaramA. Emergence of Klebsiella pneumoniae isolate co-producing NDM-1 with KPC-2 from
India.J Antimicrob Chemother. 2012;67(1):243-4. DOI: 10.1093/ $\mathrm{jac} / \mathrm{dkr} 431$ PMID: 21990048

4. PoirelL, BenoudaA, HaysC, NordmannP. Emergence of NDM1-producing Klebsiella pneumoniae in Morocco.J Antimicrob Chemother. 2011;66(12):2781-3. DOI: 10.1093/jac/dkr384 PMID: 21930570

5. WalshTR, Weeks], LivermoreDM, TolemanMA. Dissemination of NDM-1 positive bacteria in the New Delhi environment and its implications for human health: an environmental point prevalence study.Lancet Infect Dis. 2011;11(5):355-62. DOI: 10.1016/S1473-3099(11)70059-7 PMID: 21478057

6. WoodfordN, WarehamDW, GuerraB, TealeC. Carbapenemaseproducing Enterobacteriaceae and non-Enterobacteriaceae from animals and the environment: an emerging public health risk of our own making?] Antimicrob Chemother. 2014;69(2):287-91. DOI: 10.1093/jac/dkt392 PMID: 24092657

7. European Survey on Carbapenemase-Producing Enterobacteriaceae (EuSCAPE) Working Group,GlasnerC, AlbigerB, BuistG, Tambić AndrasevićA, CantonR, CarmeliY, et al. . Carbapenemase-producing Enterobacteriaceae in Europe: a survey among national experts from 39 countries, February 2013. Euro Surveill. 2013;18(28):20525. DOI: 10.2807/15607917.ES2013.18.28.20525 PMID: 23870096

8. WangX, LiH, ZhaoC, ChenH, LiuJ, WangZ, et al. Novel NDM9 metallo- $\beta$-lactamase identified from a ST107 Klebsiella pneumoniae strain isolated in China. Int J Antimicrob Agents. 2014;44(1):90-1. DOI: 10.1016/j.ijantimicag.2014.04.010 PMID: 24913967

9. HornseyM, PheeL, WarehamDW. A novel variant, NDM-5, of the New Delhi metallo- $\beta$-lactamase in a multidrug-resistant Escherichia coli ST648 isolate recovered from a patient in the United Kingdom.Antimicrob Agents Chemother. 2011;55(12):5952-4. DOI: 10.1128/AAC.05108-11 PMID: 21930874

10. SassiA, LoucifL, GuptaSK, DekhilM, ChettibiH, RolainJM. NDM5 carbapenemase-encoding gene in multidrug-resistant clinical isolates of Escherichia coli from Algeria.Antimicrob Agents Chemother. 2014;58(9):5606-8. DOI: 10.1128/AAC.02818-13 PMID: 24982080

11. PitartC, SoléM, Rocal, RománA, MorenoA, VilaJ, et al. Molecular characterization of blaNDM-5 carried on an IncFll plasmid in an Escherichia coli isolate from a nontraveler patient in Spain. Antimicrob Agents Chemother. 2015;59(1):659-62. DOI: 10.1128/AAC.04040-14 PMID: 25313215

12. WeteringsV, ZhouK, RossenJW, van StenisD, ThewessenE, Kluytmans), et al. An outbreak of colistin-resistant Klebsiella pneumoniae carbapenemase-producing Klebsiella pneumoniae in the Netherlands (July to December 2013), with inter-institutional spread. Eur J Clin Microbiol Infect Dis. 2015;34(8):1647-55. DOI: 10.1007/S10096-015-2401-2 PMID: 26067658

13. ZankariE, HasmanH, CosentinoS, VestergaardM, RasmussenS, LundO, et al. Identification of acquired antimicrobial resistance genes. J Antimicrob Chemother. 2012;67(11):2640-4. DOI: $10.1093 / \mathrm{jac} / \mathrm{dks} 261$ PMID: 22782487

14. Werkgroep Infectie Preventie (WIP). Multidrug-resistant microorganisms. [Bijzonder resistente micro-organismen (BRMO)]. Leiden: WIP. Dutch. Available from: http://www.rivm. $\mathrm{nl} / \mathrm{dsresource}$ ?objectid=rivmp:46410\&type=org\&disposition $=$ inline

15. Bialek-DavenetS, CriscuoloA, AilloudF, PassetV, JonesL, Delannoy-VieillardAS, et al. Genomic definition of hypervirulent and multidrug-resistant Klebsiella pneumoniae clonal groups. Emerg Infect Dis. 2014;20(11):1812-20. DOI: 10.3201/eid2011.140206 PMID: 25341126

16. AbdallahHM, ReulandEA, WintermansBB, Al NaiemiN, KoekA, AbdelwahabAM, et al. Extended-Spectrum $\beta$-Lactamases and/ or Carbapenemases-Producing Enterobacteriaceae Isolated from Retail Chicken Meat in Zagazig, Egypt. PLoS ONE. 2015;10(8):e0136052. DOI: 10.1371/journal.pone.0136052 PMID: 26284654

17. LesterCH, OlsenSS, JakobsenL, ArpiM, FuurstedK, HansenDS, et al. Emergence of extended-spectrum $\beta$-lactamase (ESBL)producing Klebsiella pneumoniae in Danish hospitals; this is in part explained by spread of two CTX-M-15 clones with multilocus sequence types 15 and 16 in Zealand. Int J Antimicrob Agents. 2011;38(2):180-2. DOI: 10.1016/j. ijantimicag.2011.03.018 PMID: 21612893 